\title{
BIOMASSA E FLORÍSTICA EM FLORESTAS SECUNDÁRIAS DE DIFERENTES IDADES
}

\author{
Marlete Moreira de Sousa Mendes ${ }^{1}$, Maria Elizabete de Oliveira ${ }^{2}$ \\ 'Bióloga, M.Sc, Depto. de Ciências do Solo, UFC, Fortaleza, CE, Brasil - mendes758@ hotmail.com \\ ${ }^{2}$ Agrônoma, Dra., Depto. de Zootecnia, UFPI, Teresina, PI, Brasil - maeliz@uol.com.br \\ Recebido para publicação: 17/11/2009 - Aceito para publicação: 17/05/2010
}

\begin{abstract}
Resumo
Este trabalho foi desenvolvido no município de União, PI, localizado na região centro-norte do estado, e tem como objetivo determinar a produção de biomassa e a composição florística em matas secundárias com diferentes idades após o cultivo agrícola tradicional (um, três e cinco anos de pousio, com dois tratamentos para cada idade). Para análise da vegetação, foram estabelecidas 20 parcelas em cada idade de pousio. Para levantamento das espécies arbóreas/arbustivas, foram usadas parcelas com $25 \mathrm{~m}^{2}$ e, dentro destas, subparcelas com $2,0 \mathrm{~m}^{2}$ para as espécies herbáceas. Os resultados mostram 100 espécies ocorrentes nas áreas, sendo 42 de porte arbustivo/arbóreo e 58 herbáceas. As capoeiras com um, três e cinco anos apresentaram, respectivamente, 3,$4 ; 6,0$ e 6,7 ton.ha $^{-1}$ de massa seca. Palavras-chave: Sucessão secundária; pousio; massa seca; levantamento florístico.
\end{abstract}

\begin{abstract}
Biomass production and floristic of secondary forests of different ages. This study was developed in the Union county, localized in center-north region of the Piaui State, and aim to determine the production of biomass and floristic composition of secondary forests with different ages after slash and burn agriculture (one, three and five years-old regeneration stages, with two replicates). For analysis of vegetation they were established 20 plots by each different age of fallow. Plots were used with areas of $25.0 \mathrm{~m}^{2}$ by shrub/tree and, within these, subplots with $2.0 \mathrm{~m}^{2}$ were allocated for the herbaceous species. The results show that 100 species occur in the area, 42 shrubs and 58 herbaceous species; the secondary forest with one, three and five years present total biomass of 3.4; 6.0 and 6.7 ton $\mathrm{ha}^{-1}$ of total dry mass per hectare, respectively.

Keywords: Secondary succession; fallow; dry mass; floristic survey; frequency.
\end{abstract}

\section{INTRODUÇÃO}

Em países tropicais, é grande o número de produtores que praticam a agricultura itinerante, cujo preparo da terra envolve o corte e a queima da vegetação. Na sequência é feito o plantio na área queimada, aproveitando-se os nutrientes disponíveis nas cinzas. Dois a três anos depois, os nutrientes tornam-se escassos e as plantas invasoras tornam-se um problema. Os produtores, então, abandonam o local por 10 a 20 anos, permitindo às espécies locais reflorestarem a área (sucessão secundária). Dessa forma, é necessário desmatar e limpar outras áreas para realizar novos cultivos, repetindo o processo.

As florestas secundárias podem ser definidas como vegetação sucessional, que se regenera após a vegetação inicial ter sido removida por ação antrópica (FAO, 2002). Tal vegetação provê uma combinação de árvores, arbustos e ervas, sendo geralmente instável e apresentando estádios sucessionais. Como a restauração da floresta ao seu estado original é muito difícil de ocorrer, a biomassa associada com pastagens e florestas secundárias em desenvolvimento é sempre menor que a da floresta original (BUSCHBACHER; UHL; SERRÃO, 1988).

O acúmulo de biomassa em florestas secundárias mostra um modelo de rápida acumulação em até 15 a 20 anos de pousio, contudo a taxa de incremento de biomassa está inversamente relacionada com o tempo de uso agrícola (HUGGES et al., 1992). Florestas secundárias em Porto Rico apresentam valores de biomassa similares à floresta madura somente após 40 anos de pousio (AIDE et al., 2000). Por outro 
lado, estudos mostram o potencial para a rápida recuperação da diversidade de plantas lenhosas e da estrutura em florestas secundárias adjacentes à floresta madura (MARTIN; SHERMAN; FAHEY, 2004).

Em florestas tropicais, a agricultura do tipo tradicional ou itinerante tem importante impacto local sobre o ciclo de nutrientes, organismos no solo, sementes e tudo aquilo que possa ter efeitos na qualidade do local e no reflorestamento (EWEL et al., 1980). No Nordeste do Brasil, informações sobre a ecologia de florestas secundárias são escassas, todavia, dada a ocorrência de muitas áreas e em diferentes ecossistemas, faz-se necessário gerar conhecimento sobre o assunto. Assim, este estudo objetiva determinar a composição florística da vegetação e quantificar a biomassa a ela associada, em áreas utilizadas pela agricultura itinerante com diferentes idades. Nossa hipótese é que a biomassa aumenta linearmente até cinco anos e que a composição florística aumenta em riqueza de espécies com o aumento do pousio.

\section{MATERIAIS E MÉTODOS}

\section{Área de estudo}

Este estudo foi desenvolvido no ano 2003, a $56 \mathrm{~km}$ da capital (Teresina), na área rural do município de União, localizado na região subúmida do Piauí, Brasil $\left(04^{\circ} 35^{\prime} 09^{\prime}\right.$ ' S, 4251'51” W) (Figura 1). A localidade apresenta duas estações principais: uma chuvosa, de dezembro a maio, e outra seca, de junho a novembro, com média anual de precipitação de $1400 \mathrm{~mm}$ e média anual de temperatura variando entre $36^{\circ} \mathrm{C}$ (máxima) e $21^{\circ} \mathrm{C}$ (mínima). A região é predominantemente plana, com altitude de 78 a 65 m acima do nível do mar (LIMA, 1982). Os solos são podzólicos (EMBRAPA/SUDENE/DRH, 1993), com pH variando de 5,3 a 5,7 (acidez média). Os níveis de alumínio são baixos. A vegetação apresenta fisionomia de Floresta Secundária Mista associada com palmeiras de babaçu (CODEVASF, 2006).

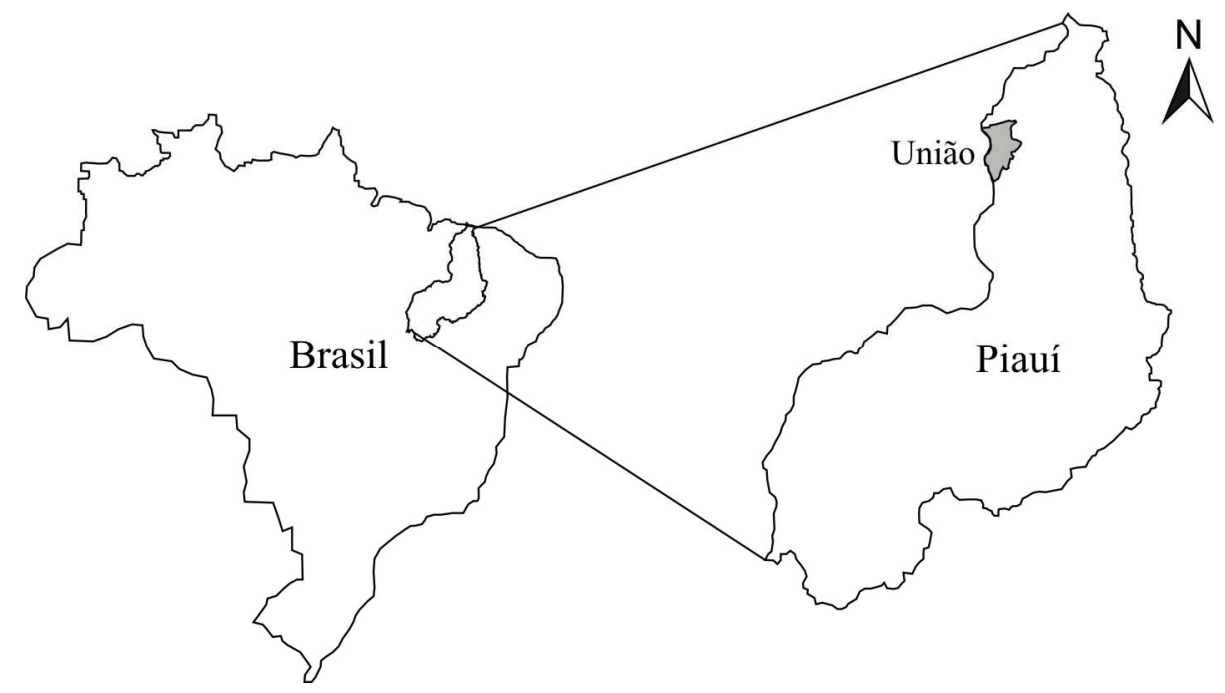

Figura 1. Localização da área de estudo.

Figure 1. Location of the study area.

\section{Quantificação da biomassa e levantamento florístico}

Foram escolhidas duas áreas com um, três e cinco anos após uso agrícola, situadas nas localidades Pedra de Fogo e Caraúba, região Nordeste do município. Cada idade da capoeira, que corresponde à idade de rebrota, foi considerada como um tratamento. Foram demarcadas 10 parcelas por tratamento, usando estacas e cordão plástico, medindo cada uma 5 x $5 \mathrm{~m}$ para árvores/arbustos (acima de um metro de altura) e, dentro destas, parcelas de 1 × $2 \mathrm{~m}$, para espécies subarbustivas e herbáceas (até um metro de altura). As parcelas foram separadas à distância de, aproximadamente, 10 metros uma da outra, em uma sequência diagonal nas áreas escolhidas. 
Em cada parcela, as plantas dos dois estratos foram cortadas ao nível do solo e pesadas, para se obter o peso total. Amostras foram separadas para pré-secagem, duas para arbustos/árvores e uma mista para as herbáceas. Estas foram pesadas, para se obter a massa total. As amostras foram conduzidas ao laboratório do Centro de Ciências Agrícolas da Universidade Federal do Piauí, retiradas da estufa após 72 horas a $70{ }^{\circ} \mathrm{C}$ e pesadas novamente, para determinação da massa seca. A disponibilidade de massa seca foi analisada através de delineamento inteiramente casualizado, com três tratamentos e 20 repetições. As médias foram testadas ao nível de 5\% de probabilidade, através do teste SNK (Kruskal-Wallis).

Foram retiradas amostras da vegetação existente nas parcelas para identificação, usando-se literatura especializada e por comparação com espécimes do Herbário Graziela Barroso (TEGB), da Universidade Federal do Piauí. Os dados e materiais foram coletados durante a estação chuvosa (março e abril), quando as plantas tinham mais folhas, uma vez que muitas delas são decíduas na estação seca.

Para o cálculo da similaridade, foi usada a fórmula: IS $=2 . c / a+b$, onde IS representa o Índice de Similaridade de Sörensen, 'a' e 'b' as idades de pousio 1 e 2 , respectivamente, e 'c' é o número de espécies comuns nas duas áreas. $\mathrm{O}$ índice varia de zero a um. Valores próximos a um indicam alta similaridade entre as áreas. Para calcular a frequência relativa (percentual de parcelas em que as espécies foram encontradas em cada capoeira), foi usada a fórmula $F R=(p \div n) \times 100$, onde $\mathrm{p}$ é o número de parcelas ocupadas pela espécie e n é o número total de parcelas instaladas. Para verificar se há correlação positiva entre idade da capoeira (variável independente) e produção de biomassa (variável dependente), foi feita regressão linear. O mesmo foi feito para correlacionar idade da capoeira (variável independente) e riqueza de espécies (variável dependente).

\section{RESULTADOS}

\section{Produção de biomassa}

A massa seca das plantas herbáceas decresce com o aumento da idade da capoeira, partindo de valores próximos a $1000 \mathrm{~kg} \cdot \mathrm{ha}^{-1}$ nas áreas com um ano de pousio, para menos de $600 \mathrm{~kg} \cdot \mathrm{ha}^{-1}$ nas capoeiras de cinco anos. Todavia a biomassa arbustiva aumentou com a idade do pousio, produzindo cerca de $2400 \mathrm{~kg} \cdot \mathrm{ha}^{-1}$ com um ano para mais de $6100 \mathrm{~kg} \cdot \mathrm{ha}^{-1}$ aos cinco anos. A massa seca total (ervas + arbustos) seguiu a mesma tendência da massa seca arbustiva, indo de valores próximos a $3.400 \mathrm{~kg} \cdot \mathrm{ha}^{-1}$ para mais de $6.700 \mathrm{~kg} \cdot \mathrm{ha}^{-1}$, conforme aumentam os anos de pousio (Figura 2).

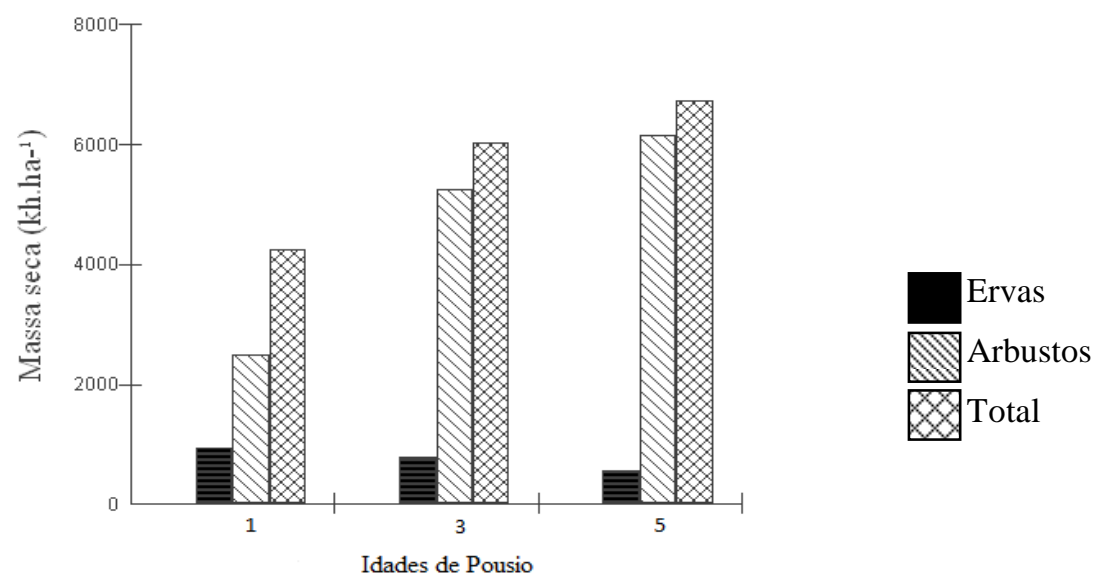

Figura 2. Massa seca total, arbustiva e herbácea em florestas secundárias de diferentes idades.

Figure 2. Total, shrubs and herbs dry mass in secondary forest of different ages.

A produção de massa seca total diferiu entre as capoeiras com um e três anos $(\mathrm{p}=0,0061)$ e entre um e cinco anos $(p=0,0474)$, mas não entre as capoeiras de três e cinco anos $(p=0,4470)$, pelo teste Kruskal-Wallis. Os valores de massa seca nas três capoeiras apresentam tendência de aumento com a idade (Figura 3). 


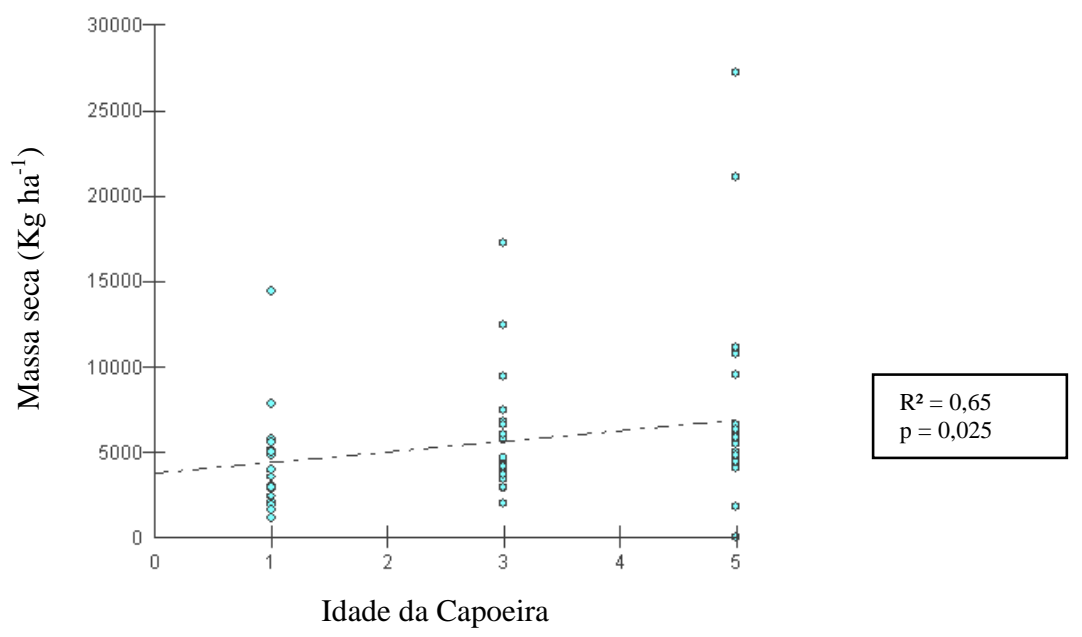

Figura 3. Relação entre idade de capoeira e produção de massa seca total em florestas secundárias de diferentes idades.

Figure 3. Relation between age of farmyard and production of total dry mass in secondary forest of different ages.

\section{Levantamento florístico}

Foram encontradas 100 espécies vegetais, sendo 42 arbustivas e 58 herbáceas, cuja distribuição está apresentada na figura 4. Desse total, 28 espécies são comuns às três áreas. O Índice de Similaridade de Sörensen (IS) mostra similaridade média entre as amostras: 0.64 entre um e três anos e 0.60 entre as capoeiras com três e cinco anos de pousio. As espécies amostradas estão distribuídas em 38 famílias, sendo que as que apresentaram maior número de espécies foram Fabaceae (15 espécies), Poaceae (nove), Euphorbiaceae (sete), Cyperaceae (seis), Malvaceae e Rubiaceae (cinco cada), Amaranthaceae, Convolvulaceae, Lamiaceae, Lythraceae e Myrtaceae (três cada). As demais famílias apresentaram uma ou duas espécies.
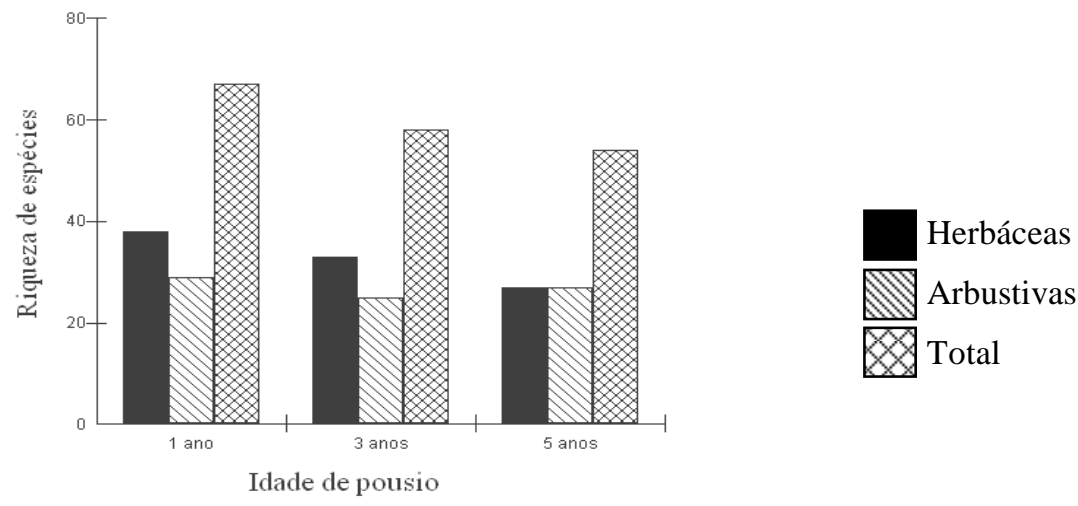

Figura 4. Número de espécies (ervas, arbustos e total) em florestas com diferentes idades de pousio. Figure 4. Number of species (herbs, shrub and total) in forests with different ages of fallow.

As espécies arbustivas e herbáceas com maior frequência relativa, considerando as capoeiras separadamente e uma média das três idades, estão na tabela 1. Dentre as arbustivas, Senna alata (L.) Roxb. é a espécie com maior frequência, estando presente nas três capoeiras, em $80 \%$ das parcelas da capoeira com três anos de pousio e, em média, ocupando 51,6\% do total de parcelas. Dentre as herbáceas, Sida sp. é a espécie mais comum, estando presente em quase 100\% das parcelas na capoeira com um ano e ocupando, em média, $73 \%$ do total de parcelas. As ciperáceas e gramíneas (Cyperaceae e Poaceae) 
diminuíram o número de espécies entre as capoeiras com um e cinco anos de pousio. A riqueza das espécies arbustivas, em relação à idade da capoeira, ajustou-se a um modelo linear (Figura 5), diminuindo com o aumento da idade de pousio.

Tabela 1. Lista das espécies arbustivas e herbáceas mais frequentes em capoeiras com diferentes idades.

Table 1. List of shrubs and herbaceous most frequent in secondary forest of different ages.

\begin{tabular}{|c|c|c|c|c|c|c|}
\hline \multirow[b]{2}{*}{ Família } & \multirow[b]{2}{*}{ Espécie } & \multicolumn{4}{|c|}{ Frequência Relativa (\%) } & \multirow[b]{2}{*}{ Hábito } \\
\hline & & $\begin{array}{c}1 \\
\text { ano }\end{array}$ & $\begin{array}{c}\mathbf{3} \\
\text { anos }\end{array}$ & $\begin{array}{c}5 \\
\text { anos }\end{array}$ & Média & \\
\hline Amaranthaceae & Alternanthera brasiliana (L.) Kuntze & 18,0 & 7,0 & 10,0 & 11,7 & Herbáceo \\
\hline Arecaceae & Orbignia phalerata Mart. & 55,0 & 50,0 & 5,0 & 36,7 & Arbustivo \\
\hline Combretaceae & Combretum leprosum Mart. & 25,0 & 25,0 & 30,0 & 26,7 & Arbustivo \\
\hline Convolvulaceae & Ipomoea bahiensis Willd. ex Roem. \& Schult. & 17,0 & 14,0 & 14,0 & 15,0 & Herbáceo \\
\hline Cyperaceae & Cyperus diffusus Vahl & 45,0 & 7,0 & 7,0 & 19,7 & Herbáceo \\
\hline \multirow[t]{2}{*}{ Euphorbiaceae } & Croton sonderianus Müll. Arg. & 45,0 & 25,0 & 30,0 & 33,3 & Arbustivo \\
\hline & Phyllantus niruri L. & 45,0 & 35,0 & 10,0 & 30,0 & Herbáceo \\
\hline \multirow[t]{5}{*}{ Fabaceae } & Bauhinia forficata Link & 25,0 & 15,0 & 35,0 & 25,0 & Arbustivo \\
\hline & Caesalpinia leiostachya (Benth.) Ducke & 15,0 & 50,0 & 50,0 & 38,3 & Arbustivo \\
\hline & Mimosa pudica $\mathrm{L}$. & 67,0 & 14,0 & 14,0 & 31,7 & Herbáceo \\
\hline & Senna alata (L.) Roxb. & 50,0 & 85,0 & 20,0 & 51,6 & Arbustivo \\
\hline & Senna obtusifolia (L.) H.S. Irwin \& Barneby & 33,0 & 28,0 & 43,0 & 34,7 & Herbáceo \\
\hline Lamiaceae & Marsypianthes chamaedrys (Vahl) Kuntze & 17,0 & 14,0 & 28,0 & 19,7 & Herbáceo \\
\hline Lythraceae & Cuphea calophylla Cham. \& Schltdl. & 14,0 & 21,0 & 24,0 & 19,7 & Herbáceo \\
\hline Malvaceae & Sida sp. & 95,0 & 50,0 & 75,0 & 73,3 & Herbáceo \\
\hline \multirow[t]{2}{*}{ Myrtaceae } & Campomanesia sp. & 15,0 & 75,0 & 50,0 & 46,7 & Arbustivo \\
\hline & Myrtus communis L. & 35,0 & 5,0 & 35,0 & 25,0 & Arbustivo \\
\hline Oxalidaceae & Oxalis diffusa Pohl ex Progel & 25,0 & 7,0 & 17,0 & 16,3 & Herbáceo \\
\hline \multirow[t]{2}{*}{ Poaceae } & Brachiaria fasciculata (Sw.) Parodi & 45,0 & 35,0 & 17,0 & 32,3 & Herbáceo \\
\hline & Panicum laxum Sw. & 45,0 & 14,0 & 21,0 & 26,7 & Herbáceo \\
\hline
\end{tabular}

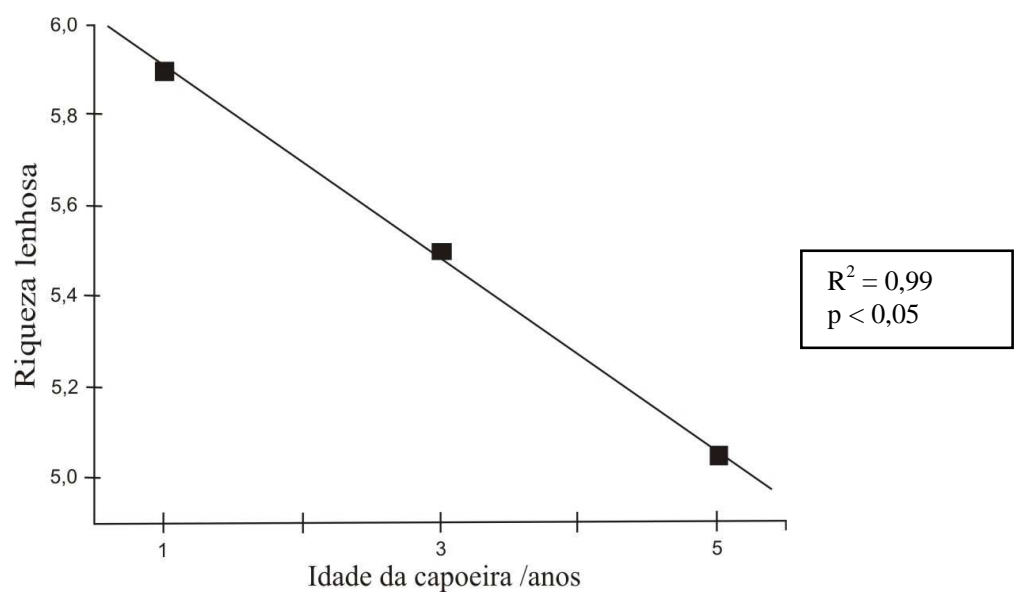

Figura 5. Riqueza de espécies em capoeiras com um, três e cinco anos de pousio.

Figure 5. Species richness in secondary forest with one, three and five years of fallow.

\section{DISCUSSÃO}

A massa seca das plantas herbáceas diminui com o aumento da idade de pousio, provavelmente devido à diminuição de espécies e espécimes herbáceos. Com o crescimento dos arbustos, aumenta o sombreamento sobre as ervas, impedindo o seu desenvolvimento. Cheung (2006) observou, em áreas usadas para pastagem na Floresta Atlântica, o aumento na biomassa herbácea após o abandono, seguido 
pela diminuição ao longo do tempo. Em regiões semiáridas, os resultados são similares, com a biomassa herbácea diminuindo à medida que aumenta o pousio (BEHERA; MISRA, 2006).

O acúmulo de fitomasssa do estrato lenhoso é maior no intervalo entre um e três anos, observando-se um aumento superior a $100 \%$. A diferença de acúmulo entre os anos três e cinco é apenas de $703 \mathrm{~kg}$, correspondendo a um aumento de $11 \%$. Esses resultados podem estar associados a uma maior densidade das plantas por área, o que resulta em aumento de competição por água, luz e nutrientes, com redução da produção de biomassa (BRIENZA-JÚNIOR, 1999). Cardoso et al. (2000) afirmam que, com o aumento de queimadas na área, diminui a quantidade de biomassa, pois uma área preparada para o cultivo com o uso do fogo apresenta até $36 \%$ a menos de biomassa quando comparada a uma em que não houve utilização de fogo. Esse valor pode subir para $50 \%$ se houver uma segunda queimada na área.

Nas capoeiras de matas de babaçu, a produção de biomassa foi inferior à encontrada por pesquisadores em outras regiões do país. Nunez (1995) observou produção de 22 toneladas de massa verde em capoeiras de quatro anos de idade na Amazônia, enquanto Vielhauer et al. (1998) observaram, também na Amazônia, uma produção de nove toneladas de massa seca em capoeiras com 12 meses de pousio. Nas capoeiras aqui estudadas, aos cinco anos a produção por hectare de matéria verde é cerca de 12 toneladas, e a de matéria seca é, em média, de seis toneladas. Convém ressaltar que as capoeiras objeto deste trabalho estão abertas ao pastejo por animais, geralmente em situação de altas lotações, o que deve resultar em uma redução de biomassa. Considerando ainda a Amazônia como área de estudo, nota-se que a vegetação secundária se restabelece bem em até sete anos (GAMA et al., 2002), enquanto que em mata de babaçu são necessários mais anos para que a vegetação assemelhe-se à original.

Neste trabalho, a tendência foi de queda da riqueza de espécies com a distância do distúrbio. Partindo de 65 espécies (entre herbáceas e arbustivas/arbóreas) em capoeiras com um ano de pousio para 52 espécies aos cinco anos (Figura 3), a principal baixa ocorreu entre as ervas. Resultados semelhantes foram observados na Amazônia, com maior número de espécies nos anos iniciais da sucessão e um menor número aos 40 anos (FERREIRA; PRANCE, 1999). De acordo com Cheung (2006), quanto maior o tempo pós-distúrbio, maior seria também a diversidade da área se a trajetória sucessional esperada fosse sempre um padrão gradual de incremento de espécies. Sampaio et al. (1998) confirmam que há diminuição do número de espécimes vegetais em Caatinga quando se utiliza o corte seguido de queima, sendo que menos de 50\% rebrotam num período de até dois meses. Todavia, estudos sugerem que o fogo pode acelerar o desenvolvimento de algumas espécies vegetais (BREWER et al., 2009) ou não ter impacto negativo sobre a quantidade de nutrientes disponíveis (VOURLITIS; PASQUINI, 2008). Capitanio; Carcaillet (2008) observaram que a riqueza nos dois anos seguintes ao incêndio apresenta os maiores valores, mas são semelhantes aos valores para áreas com até 30 anos após o fogo, e que a diversidade de espécies vegetais assume valores semelhantes entre a floresta com dois anos de pousio e uma área sem manejo há 80 anos.

Ceccon et al. (2002) assinalam que algumas propriedades do solo podem afetar o número de indivíduos e a riqueza de espécies das comunidades de árvores e de plântulas. De acordo com Armesto; Pickett (1985), há uma relação direta entre a intensidade do distúrbio causado pela agricultura e a riqueza de espécies: quanto maior o distúrbio causado, menor a riqueza de espécies.

Os resultados sugerem que a resiliência de capoeiras em matas de babaçu é baixa em comparação com outros ecossistemas. Todavia mais pesquisas são necessárias sobre sucessão secundária nesse ecossistema que possam precisar o tempo para restaurar a floresta. Os efeitos do fogo sobre a vegetação permanecem incertos e, para quantificar as perdas pela agricultura de broca-e-queima sobre a diversidade e produção de matéria orgânica, são necessárias pesquisas usando uma área de floresta intacta, cujos resultados possam ser comparados com os valores aqui apresentados. Conclui-se que, aos cinco anos, a biomassa ainda é baixa, sendo necessário mais tempo para restaurar a floresta, uma vez que a biomassa dirige a cronossequência de aumento no estoque de carbono no ecossistema (KIYONO et al., 2007), necessário ao desenvolvimento da vegetação.

\section{CONCLUSÃO}

A biomassa e diversidade vegetal apresentam baixos valores em cinco anos de pousio. A riqueza de espécies diminui com o aumento do pousio e o aumento da biomassa total é principalmente em função da arbustiva, uma vez que ocorre diminuição na biomassa herbácea. Essa pesquisa mostra que tais atributos, essenciais à recuperação de uma área que foi desmatada e submetida à queima, ainda estão em déficit aos cinco anos de pousio, sendo, assim, necessário mais tempo para que a área esteja recuperada. 


\section{REFERÊNCIAS}

AIDE, T. M.; ZIMMERMAN, J. K.; PASCARELLA, J. B.; RIVERA, L.; MARCANO-VEGA, H. Forest regeneration in a chronosequence of tropical abandoned pastures: implications for restoration ecology. Restoration Ecology, Perth, v. 8, n. 4, p. 328-338, 2000.

ARMESTO, J. J.; PICKETT, S. T. Experiments on disturbance in old-field plant communities: impact on species richness and abundance. Ecology, Ithaca, n. 66, v. 1, p. 230-240, 1985.

BEHERA, S. K.; MISRA, M. K. Floristic and structure of the herbaceous vegetation of four recovering forest stands in the Eastern Ghats of India. Biodiversity and Conservation, New York, v. 15, n. 7, p. 2263-2285, 2006.

BREWER, J. S.; CUNNINGHAM, A. L.; MOORE, T. P.; BROOKS, R. M.; WALDRUP, J. L. A sixyear study of fire-related flowering cues and coexistence of two perennial herbs in a wet longleaf pine (Pinus palustris) savanna. Plant Ecology, New York, v. 200, n. 1, p.141-154, 2009.

BRIENZA-JÚNIOR, S. Biomass dynamics of fallow vegetation enriched with leguminous trees in the Eastern Amazon of Brazil. Goettingen: Verlag Erich Goltze Gmbh, 1999. v. 1. 133 p.

BUSCHBACHER, R.; UHL, C.; SERRÃO, A. S. Abandoned pastures in eastern Amazonia. II. Nutrient stocks in the soil and vegetation. Journal of Ecology, Oxford, v. 76, n. 3, p. 682-699, 1988.

CAPITANIO, R.; CARCAILlET, C. Post-fire Mediterranean vegetation dynamics and diversity: A discussion of succession models. Forest, Ecology and Management, Maryland Heights, v. 255, n. 3-4, p. 431-439, 2008.

CARDOSO, E. L.; CRISPIM, S. M. A.; RODRIGUES, C. A. G.; BARIONI-JÚNIOR, W. Biomassa aérea e produção primária do estrato herbáceo em campo de Elyonurus muticus submetido a queima anual, no pantanal. Pesquisa Agropecuária Brasileira, Brasília, v. 35, n. 8, p. 1501-1507, 2000.

CECCON, E.; OLMSTED, I.; ALVES, J. C. Vegetation and soil properties in two tropical dry forests of differing regeneration status in Yucatan. Agrociência, Pelotas, v. 36, n. 5, p. 621-631, 2002.

CHEUNG, K. C. Regeneração natural em áreas de Floresta Atlântica na Reserva Natural Rio Cachoeira, Antonina, PR. 2006. 69 p. Dissertação (Mestrado em Engenharia Florestal) - Universidade Federal do Paraná, Curitiba, 2006.

CODEVASF. Companhia de Desenvolvimento dos Vales do São Francisco e do Parnaíba. In: Plano de ação para o desenvolvimento integrado da Bacia do Parnaíba. Brasília: TDA Desenho \& Art Ltda., 2006.

EMBRAPA/SUDENE/DRH. Solos do Piauí. Teresina: EMBRAPA, 1993.

JOHN EWEL, J.; BERISH, C.; BROWN, B.; PRICE, N.; RAICH, J. Slash and burn impacts on a Costa Rican wet forest site. Ecology, Ithaca, v. 62, n. 3, p. 816-829, 1980.

FAO. Workshop on tropical secondary forest management in Africa: realities and perspectives. FAO, 2002. Disponível em: 〈www.fao.org/DOCREP>. Acesso em 20 jan 2004.

FERREIRA, L. V.; PRANCE, G. T. Ecosystem recovery in terra firm forests after cutting and burning: a comparison on species richness, floristic composition and forest structure in the Jaú National Park, Amazonia. Botanical Journal of the Linnean Society, Londres, v. 130, n. 2, p. 97-110, 1999.

GAMA, J. R. V.; BOTELHO, S. A.; BENTES-GAMA, M. M. Composição florística e estrutura da regeneração natural de floresta secundária de várzea baixa no estuário amazônico. Árvore, Viçosa, v. 26, n. 5 , p. 559-566, 2002.

HUGHES, R. F.; KAUFFMAN, J. B.; JARAMILLO, V. J. Biomass, carbon and nutrient dynamic of secondary forests in a humid tropical region of Mexico. Ecology, Ithaca, v. 80, n. 6, p. 1892-1907, 1992.

KIYONO, Y.; OCHIAI, Y.; CHIBA, Y.; ASAI, H.; SAITO, K.; SHIRAIWA, T.; HORIE, T.; SONGNOUKHAI, V. Predicting chronosequential changes in carbon stocks of pachymorph bamboo 
communities in slash-and-burn agricultural fallow, northern Lao People's Democratic Republic. Journal of Forest Research, Bunkyo-ku, v. 12, n. 5, p. 371-381, 2007.

LIMA, M. G. Critérios climatológicos para delimitação do semiárido no estado do Piauí. Ciências Agrárias, Teresina, v. 1, n. 1, p. 33-61, 1982.

MARTIN, P. H.; SHERMAN, R. E.; FAHEY, T. J. Forty years of tropical forest recovery from agriculture: structure and floristics of secondary and old-growth riparian forests in the Dominican Republic. Biotropica, Zurich, v. 36, n. 3, p. 297-317, 2004.

NUNEZ, J. B. H. Biomassa e estoque de bioelementos das diversas fases da vegetação secundária, provenientes de diferentes sistemas de uso da terra no nordeste paraense, Brasil. Belém: UFPA, 1995.

SAMPAIO, E. V. S. B.; ARAÚJO, E. L.; SALCEDO, I. H.; TIESSEN, H. Regeneração da vegetação de Caatinga após corte e queima, em Serra Talhada, PE. Pesquisa Agropecuária Brasileira, Brasília, v. 33, n. 5, p. 621-632, 1998.

VIELHAUER, K.; SÁ, T. D. de A.; KANASHIRO, M.; DENICH, M. Technology development of slashand-mulch and of fallow enrichment in shifting cultivation systems of the Eastern Amazon. In: SHIFT Proceedings of the third SHIFT, 3., 1998, Manaus. Workshop. Manaus: Embrapa, 1998. p. 49-59.

VOURLITIS, G. L.; PASQUINI, S. C. Carbon and nitrogen dynamics of pre- and post-fire chaparral exposed to varying atmospheric $\mathrm{N}$ deposition. Journal of Arid Environments, Exeter, v. 72, n. 8, p. 1448-1463, 2008. 